Research Article

\title{
Vibration Modes and Parameter Analysis of V-Shaped Electrothermal Microactuators
}

\author{
Zhuo Zhang, ${ }^{1}$ Yueqing Yu, ${ }^{1}$ and Xuping Zhang ${ }^{2}{ }^{2}$ \\ ${ }^{1}$ College of Mechanical Engineering and Applied Electronics Technology, Beijing University of Technology, Beijing 100124, China \\ ${ }^{2}$ Department of Engineering, Aarhus University, 8000 Aarhus C, Denmark \\ Correspondence should be addressed to Xuping Zhang; xuzh@eng.au.dk
}

Received 13 December 2017; Revised 26 June 2018; Accepted 8 July 2018; Published 12 August 2018

Academic Editor: Vadim V. Silberschmidt

Copyright (c) 2018 Zhuo Zhang et al. This is an open access article distributed under the Creative Commons Attribution License, which permits unrestricted use, distribution, and reproduction in any medium, provided the original work is properly cited.

Comprehensive analysis on the modal characteristics of $\mathrm{V}$-shaped electrothermal microactuators is presented in this paper for the first time. Considering the unique geometric characteristics of the $\mathrm{V}$-shaped beam, that is, two inclined beams supporting a movable shuttle, both the lateral and longitudinal deflections are taken into account in the modal analysis. Boundary and continuity conditions are employed to obtain the frequency equation. Natural frequencies are then obtained by solving the frequency equation. Mode shapes corresponding to their natural frequencies are also calculated analytically. The theoretical modal analysis is verified with the finite element analysis using ANSYS software. Based on the model analysis, this paper further investigates the relationship between natural frequencies and the volume scaling of the $\mathrm{V}$-shaped beam. Finally, comprehensive parametric studies in terms of material properties and structural dimensions are conducted to provide insights and guidance in designing the $\mathrm{V}$-shaped beam electrothermal microactuators.

\section{Introduction}

Electrothermal microactuators have been developed for a variety of applications in MEMS (microelectromechanical systems) including RF switches [1], micropumps [2], microgrippers $[3,4]$, nanopositioners $[5,6]$, and microtesting devices [7]. Compared to other types of actuation mechanisms such as electrostatic [8], electromagnetic [9], and piezoelectric [10], electrothermal microactuators work on the thermal expansions of beam structures and have been demonstrated to be compact, stable, and large displacement and force techniques $[11,12]$. Various electrothermal actuators have been demonstrated up to date in achieving inplane $[11,13]$ and out-of-plane [14-16] motion.

Restricted by the current bulk micromachining process, it is difficult, if not impossible, to fabricate an out-of-plane microactuator with three-dimensional features. Therefore, most out-of-plane electrothermal actuators are designed based on existing in-plane actuator structures [17]. In fact, in-plane actuators are most extensively used in MEMS devices compared to the out-of-plane actuators. The U$[18,19], \mathrm{V}-[20,21]$, and Z-shaped [22-24] beams are the most fundamental actuator types in achieving the in-plane motion. A variety of structures that have been proposed are mainly based on these three types of beams.

The U-shaped beam electrothermal microactuators are capable of producing an arc circular motion by working on the difference of the thermal expansions between the thin and the thick beams. The U-shaped beam actuators are usually used as the microgripper $[18,25]$ or in MEMS positioning systems [26]. Because the thin beam has to overcome the thick beam to produce a motion and force, the $\mathrm{U}$-shaped beam outputs smaller displacement and force compared to the V-and Z-shaped beams [27]. In addition, the U-shaped actuators inevitably undergo an unwanted overshoot in reaching the steady state [27]. The V- and $\mathrm{Z}$-shaped beam actuators, on the contrary, comprise several pairs of symmetric $\mathrm{V}$-/Z-shaped beams supporting a middle shuttle and being fixed at two ends. When applying an electrical potential difference on the two ends, the beams will expand thus pushing the shuttle forward and producing motion and force. Since those pairs of beams are symmetrically configured, the actuators with V- or Z-shaped beams can generate rectilinear motion, and there is no 
overshoot during operations. This rectilinear motion facilitates the development of more complex devices by combining the V- or Z-shaped actuators with compliant mechanisms in achieving more flexible functions or higher performances $[28,29]$. Therefore, in recent decades, these two types of beams have attracted extensive research efforts. Although the V-and Z-shaped beams are demonstrated to behave similarly in electrothermal actuation, the stiffness of the Z-shaped beam is much smaller compared to that of the $\mathrm{V}$-shaped beam [30, 31]. As a result, the Z-shaped beams are often used for bidirectional actuations [32, 33].

A number of research efforts have been devoted in static modelling [34], design [35, 36], and fabrication [37] of the $\mathrm{V}$-shaped beams. The V-shaped beams are demonstrated to be capable of producing large displacement and force. To the best knowledge of the authors, few research efforts have been made in dynamics of the $\mathrm{V}$-shaped beams. In the our previous preliminary work $[27,38]$, they have established a dynamic model of the $\mathrm{V}$-shaped actuators in both air and vacuum environments to describe the dynamic behavior of the V-shaped beams. However, this model takes into account only the electrothermal response, and the thermomechanical part is treated as pseudostatic with the assumption that the mechanical frequency is much higher compared to the electrothermal response. This model is problematic when the mechanical frequency decreases with increased beam length. In this case, the mechanical vibration cannot be omitted if more accurate dynamic control is required to be achieved. Research efforts have been made to partially address this problem. For instance, Burnie [39] has calculated the first-order natural frequencies of the V-shaped beam by treating the $\mathrm{V}$-shaped beam as a spring. However, this oversimplification results in 7.2 percent derivations compared to simulated results. In addition, this does not allow one to compute higher-order frequencies and the related mode shapes. Stokey [40] has studied the free vibration of a pair of simply supported beams carrying a mass in the middle. This research work provides a guidance for describing the vibration of the shuttle of the V-shaped actuators. However, the beam is not inclined with an angle, and only lateral vibrations are considered based on the traditional line-shaped beam assumptions. Our preliminary work [41], a conference paper, pointed out that the V-shaped beam involves vibrations not only in the lateral direction but also in the longitudinal direction due to the existence of the inclined angle. Preliminary results showed that the errors of natural frequencies between the analytical and the simulated results are well below $2 \%$ by incorporating both the longitudinal and lateral vibrations. This demonstrates that the vibration of the V-shaped beam is essentially coupled between longitudinal and lateral directions. Furthermore, the conference paper only presented the calculation of natural frequencies. The computation of the mode shapes were only conducted numerically using finite element simulations.

A few research efforts have also reported parameter studies of the V-shaped electrothermal microactuators based on the analytical models. Zhu et al. [7, 22] and Shen and Chen [42] discussed the effect of the inclined angle and beam width on the static deflection, stiffness, and axial internal force. In our previous papers [30, 31], we provide comprehensive parameter studies on the influence of the inclined angle $(\theta)$, half span of beam $\left(L_{0}=L_{2} / \tan \theta\right)$, beam width, beam thickness, and shuttle dimensions on the static output displacement and force. For the dynamic behavior, we also calculated the relationship between rise time and beam dimensions for both vacuum and air conditions [27]. However, up to now, no research effort has been reported on the correlations between vibration frequencies and beam dimensions.

This paper aims to provide a comprehensive modal analysis and parameter study of the $\mathrm{V}$-shaped beam electrothermal microactuators. First, we establish the equations of motion in the longitudinal and lateral directions, respectively. Then, the frequency equations are derived subjected to both longitudinal and lateral constraint conditions. By solving the frequency equations, the natural frequencies and the associated mode shapes can be calculated. Analytically calculated natural frequencies and related mode shapes are compared with the results of the finite element simulations using ANSYS software to verify the presented modal analysis. With this, we extend the modal analysis of the single pair of beam to multipairs of beams. For design purposes, we also theoretically proved that the natural frequencies of the $\mathrm{V}$-shaped beam are inversely proportional to the volume scaling of the beam. Furthermore, parameter analysis is carried out to reveal the correlations between the natural frequencies and both the material properties and beam dimensions. These results provide insights on the vibration characteristic analysis and design of the $\mathrm{V}$-shaped beams structures. This paper presents the first insight study on the modal analysis and parameter studies of the V-shaped beam electrothermal actuators. The aim is at laying the foundation of conducting dynamic thermomechanical modelling, dynamic design, and control of the V-shaped electrothermal microactuators.

This paper is organized as follows. In Section 2, the free vibration of the $\mathrm{V}$-shaped beam is formulated incorporating both longitudinal and lateral deflections. The natural frequencies and the associated mode shapes are then calculated. The analytically computed natural frequencies and the mode shapes are compared with the results of finite element simulations using ANSYS software in Section 3. The modal analysis for of a single pair of beams is further extended to a multipair case. Section 4 presents the investigation into the relationship between the natural frequencies and the volume scaling of the V-shaped beams. In Section 5, we conduct detailed parameter analysis between the natural frequencies and both the material properties and the beam dimensions of the V-shaped beams.

\section{Vibration Analysis and Equations}

The structure of the V-shaped beam electrothermal microactuator is shown in Figure 1, where $\theta$ is the inclined angle, the length of the beam is measured as $L_{\mathrm{b}}$, the thickness and width of the beams are denoted as $b$ and $h_{0}$, respectively, half span of the beam is $L_{0}\left(L_{0}=L_{\mathrm{b}} \cos \theta\right)$, and $L_{\mathrm{s}}$ and $h_{1}$ represent the length and width of the shuttle, respectively. 


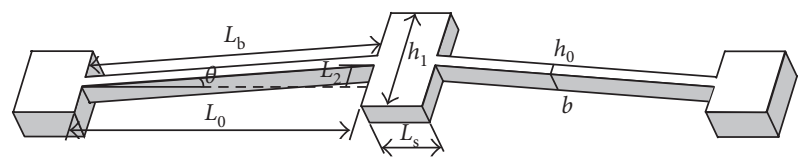

Figure 1: Schematic diagram of a V-shaped electrothermal microactuator.

When applying a voltage at the two ends, the temperature of the beam will increase due to Joule heating. As a result, the beam will expand to push the shuttle forward.

Apparently, if the shuttle is supported with a pair of traditional line-shaped beams, that is, the inclined angle $\theta$ equals to zero, the deflection of the beams is only in the lateral direction [40]. In the case of the V-shaped beam, however, due to the existence of the inclined angle, besides the lateral deflections, longitudinal beam deflections are also created for this symmetric structure. In what follows, both the longitudinal and lateral vibrations will be taken into account in the modal analysis of the V-shaped beam electrothermal microactuators. In this section, we only discuss the modal analysis for the single pair of the $\mathrm{V}$-shaped beam case, which can be easily extended to the multipairs of beam cases with the mass of the shuttle being evenly distributed for each single pair of beams.

2.1. Equations of Motion. In this section, the equations of motion are formulated in both lateral and longitudinal directions. Because of symmetry, half of the $\mathrm{V}$-shaped beam is considered, as shown in Figure 2(a), where the axis $x$ is along the longitudinal direction and the axis $y$ represents the lateral direction. $w(x, t)$ and $u(x, t)$ represent the lateral and longitudinal deflections, respectively. To derive the differential equation of motion of $\mathrm{V}$-shaped beams, all the forces and moments acting on the cross sections of a small element of the beam are taken into account, as shown in Figure 2(b). $P(x, t)$ is the axial force, $V(x, t)$ is the shear force, and $M(x, t)$ is the bending moment. In the following discussions, $\rho, E, A$, and $I$ are the density, Young's modulus, crosssectional area, and moment of inertia of the beam, respectively.

The equation of motion for the free lateral vibration can be expressed as

$$
c_{1}^{2} \frac{\partial^{4} w}{\partial x^{4}}(x, t)+\frac{\partial^{2} w}{\partial t^{2}}(x, t)=0,
$$

where $c_{1}=\sqrt{(E I / \rho A)}$. The equation of motion for the free longitudinal vibration of a uniform beam is

$$
c_{2}^{2} \frac{\partial^{2} u}{\partial x^{2}}(x, t)=\frac{\partial^{2} u}{\partial t^{2}}(x, t),
$$

where $c_{2}=\sqrt{(E / \rho)}$.

The free vibration solution can be found using the method of separation of variables as

$$
\begin{gathered}
w(x, t)=W(x) T(t), \\
u(x, t)=U(x) T(t) .
\end{gathered}
$$

Substituting (3) into (1) and (4) into (2), respectively, and rearranging lead to

$$
\begin{aligned}
U(x) & =D_{1} \cos \zeta x+D_{2} \sin \zeta x, \\
T(t) & =\widetilde{A} \cos \omega t+\widetilde{B} \sin \omega t,
\end{aligned}
$$$$
W(x)=C_{1}(\cos \beta x+\cosh \beta x)+C_{2}(\cos \beta x-\cosh \beta x)
$$$$
+C_{3}(\sin \beta x+\sinh \beta x)+C_{4}(\sin \beta x-\sinh \beta x),
$$

where $\widetilde{A}$ and $\widetilde{B}$ in (7) are constants that can be found from the initial conditions which will not be discussed in this work. $C_{1}, C_{2}, C_{3}$, and $C_{4}$ as well as $D_{1}$ and $D_{2}$ are different constants that can be found from the boundary and continuity conditions. $\beta$ and $\zeta$ relate the natural frequency $\omega$ as

$$
\begin{gathered}
\beta^{4}=\frac{\omega^{2}}{c_{1}^{2}}=\frac{\rho A}{E I} \omega^{2}, \\
\zeta=\frac{\omega}{c_{2}}=\sqrt{\frac{\rho}{E}} \omega .
\end{gathered}
$$

2.2. Boundary and Continuity Conditions. Since the equations of motion for the lateral and longitudinal vibrations in (1) and (2) involve the fourth-order and second-order derivatives with respect to $x$, respectively, six boundary or continuity conditions are required to obtain the frequency equation. The boundary and continuity conditions depict the force and deflection relationships at $x=0$ and $x=L_{\mathrm{b}}$, as listed in Table 1. The boundary conditions summarized in the first four rows in Table 1 are self-explained. The last two rows are the continuity conditions, which describe the relationship between longitudinal and lateral deflections or forces. Due to symmetry, the movement of the shuttle is confined in the $Y$ direction as shown in Figure 2(a), which require that the longitudinal and lateral deflections satisfy $u\left(L_{\mathrm{b}}, t\right) \cos \theta=w\left(L_{\mathrm{b}}, t\right) \sin \theta$, as shown in Figure 3(a). Using separation of variables, we have $U \cos \theta=$ $W \sin \theta$ at $x=L_{\mathrm{b}}$. Since the inertia force of half of the shuttle is $(m / 2)\left[\left(\partial^{2} w / \partial t^{2}\right)\left(L_{\mathrm{b}}, t\right) \cos \theta+\left(\partial^{2} u / \partial t^{2}\right)\left(L_{\mathrm{b}}, t\right) \sin \theta\right]$, where $m$ is mass of the shuttle, the force equation of motion in the direction of the movement of the shuttle at $x=L_{\mathrm{b}}$ can be given as

$$
V \cos \theta-P \sin \theta=\frac{m}{2}\left[\frac{\partial^{2} w}{\partial t^{2}}\left(L_{\mathrm{b}}, t\right) \cos \theta+\frac{\partial^{2} u}{\partial t^{2}}\left(L_{\mathrm{b}}, t\right) \sin \theta\right] .
$$

As shown in Figure 3(b), Equation (10) can be rewritten as

$$
E I \frac{\partial^{3} w}{\partial x^{3}} \cos \theta-E A \frac{\partial u}{\partial x} \sin \theta=\frac{m}{2}\left(\frac{\partial^{2} w}{\partial t^{2}} \cos \theta+\frac{\partial^{2} u}{\partial t^{2}} \sin \theta\right) \text {. }
$$

Inserting (3), (4), and (7) into (11), we obtain $2 E I \cos \theta W^{\prime \prime \prime}+m \omega^{2} \cos \theta W=2 E A \sin \theta U^{\prime}-m \omega^{2} \sin \theta U$. 


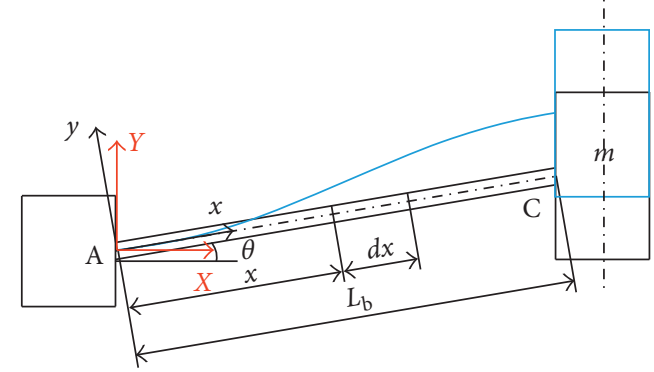

(a)

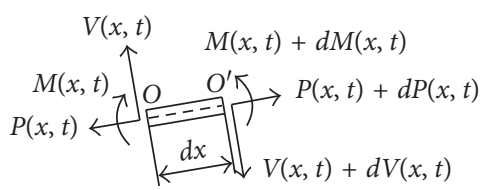

(b)

FIgURe 2: V-beam in bending.

TABLE 1: Boundary and continuity conditions.

\begin{tabular}{|c|c|c|}
\hline$x$ & $u, w$ & $U, W$ \\
\hline$x=0$ & $\begin{array}{c}u=0 \\
w=0 \\
(\partial w / \partial x)=0\end{array}$ & $\begin{array}{c}U=0 \\
W=0 \\
W^{\prime}=0\end{array}$ \\
\hline$x=L_{\mathrm{b}}$ & $\begin{array}{c}(\partial w / \partial x)=0 \\
u \cos \theta=w \sin \theta \\
V \cos \theta-P \sin \theta= \\
(m / 2)\left(\left(\partial^{2} w / \partial t^{2}\right) \cos \theta+\right. \\
\left.\left(\partial^{2} u / \partial t^{2}\right) \sin \theta\right)\end{array}$ & $\begin{array}{c}W^{\prime}=0 \\
U \cos \theta=W \sin \theta \\
2 E I \cos \theta W^{\prime \prime \prime}+m \omega^{2} \cos \theta W= \\
2 E A \sin \theta U^{\prime}-m \omega^{2} \sin \theta U\end{array}$ \\
\hline
\end{tabular}

2.3. Frequency Equation. Applying the boundary and continuity conditions into (5) and (6) leads to a system equation as in the following equation. The constants $D_{1}, C_{1}$, and $C_{3}$ in (5) and (6) equal to zero:

$$
\left(\begin{array}{lll}
a_{11} & a_{12} & 0 \\
a_{21} & a_{22} & a_{23} \\
a_{31} & a_{32} & a_{33}
\end{array}\right)\left[\begin{array}{l}
C_{2} \\
C_{4} \\
D_{2}
\end{array}\right]=0 .
$$

For the convenience of expression, the following notations are made: $s=\sin \left(\beta L_{\mathrm{b}}\right), \quad c=\cos \left(\beta L_{\mathrm{b}}\right), \quad s h=\sinh \left(\beta L_{\mathrm{b}}\right)$, $c h=\cosh \left(\beta L_{\mathrm{b}}\right), \bar{s}=\sin \left(\zeta L_{\mathrm{b}}\right)$, and $\bar{c}=\cos \left(\zeta L_{\mathrm{b}}\right)$. The elements of the coefficient matrix in (13) are $a_{11}=-s-s h, a_{12}=c-c h$, $a_{21}=(c-c h) \sin \theta, a_{22}=(s-s h) \sin \theta, a_{23}=-\bar{s} \cos \theta, a_{31}=$ $\left[2 E I(s-s h) \beta^{3}+m \omega^{2}(c-c h)\right] \cos \theta, a_{32}=\left[2 E I(-c-c h) \beta^{3}+\right.$ $\left.m \omega^{2}(s-s h)\right] \cos \theta$, and $a_{33}=\left(-2 E A \zeta \bar{c}+m \omega^{2} \bar{s}\right) \sin \theta$. Clearly, the coefficient matrix of (13) is only with respect to the natural frequency $\omega$. For nontrivial solutions of $C_{2}, C_{4}$, and $D_{2}$, the determinant of the coefficient matrix must be zero, which gives the frequency equation as follows:

$$
\begin{aligned}
m \omega^{2} \bar{s}(1-c \cdot c h)= & 2 \zeta E A \bar{c}(1-c \cdot c h) \sin ^{2} \theta \\
& +2 \beta^{3} E I \bar{s}(s \cdot c h+c \cdot s h) \cos ^{2} \theta
\end{aligned}
$$

If there is no shuttle in the middle, that is, $m=0$, the frequency equation becomes

$$
\zeta A \bar{c}(1-c \cdot c h) \sin ^{2} \theta+\beta^{3} I \bar{s}(s \cdot c h+c \cdot s h) \cos ^{2} \theta=0 .
$$

The frequency equations (14) and (15) are highly coupled, which do not allow one to obtain a simple analytical form of natural frequency $\omega$. The value of $\omega$ must then be calculated numerically. Note that the $n$th order of natural frequency is written as $\omega_{n}$.
2.4. Mode Shapes. Once the natural frequencies are obtained, the coefficient matrix of (13) is solvable. Then, it is convenient to write $C_{2}$ and $C_{4}$ with respect to $D_{2}$ as follows:

$$
\begin{aligned}
& C_{2}=-\frac{a_{12} a_{23}}{a_{12} a_{21}-a_{11} a_{22}} D_{2}, \\
& C_{4}=\frac{a_{11} a_{23}}{a_{12} a_{21}-a_{11} a_{22}} D_{2} .
\end{aligned}
$$

Substituting (16) and (17) into (5) and (6) and noticing that $D_{1}, C_{1}$, and $C_{3}$ equal to zero, the mode shapes of the lateral and longitudinal vibrations are calculated, respectively, as follows

$$
\begin{aligned}
W(x)= & k_{0} D_{2}\left[-a_{12}(\cos \beta x-\cosh \beta x)\right. \\
& \left.+a_{11}(\sin \beta x-\sinh \beta x)\right], \\
U(x)= & D_{2} \sin \zeta x,
\end{aligned}
$$

where $k_{0}=\left(a_{23} / a_{12} a_{21}-a_{11} a_{22}\right)$. It is seen that given the value of the natural frequency and $D_{2}$, the shape of the beam is determined. In contrast to the traditional line-shaped beam cases, the mode shapes of the V-shaped beam involve the coupling of the lateral and longitudinal deflections. The mode shapes can be computed as in (20) and (21) with respect to the base frame $X A Y$ in Figure $2(a) . W(x)$ and $U(x)$ are lateral and longitudinal deflections calculated with (18) and (19), respectively.

$$
\begin{aligned}
& X=x+U \cos \theta-W \sin \theta, \\
& Y=x \tan \theta+W \cos \theta+U \sin \theta,
\end{aligned}
$$

where $x \in\left(0 \sim L_{0}\right)$.

\section{Finite Element Analysis}

In this section, the natural frequencies and associated mode shapes of the $\mathrm{V}$-shaped beams are calculated analytically and compared with finite element analysis using ANSYS software (version, 14.0). The 3-D SOLID227 element is utilized to allow for the three-dimensional modelling and analysis of the V-shaped beam. A set of beam dimensions as well as material properties used in calculations and simulations are listed in Table 2. Note that, in the preceding section, $\omega_{n}$ 


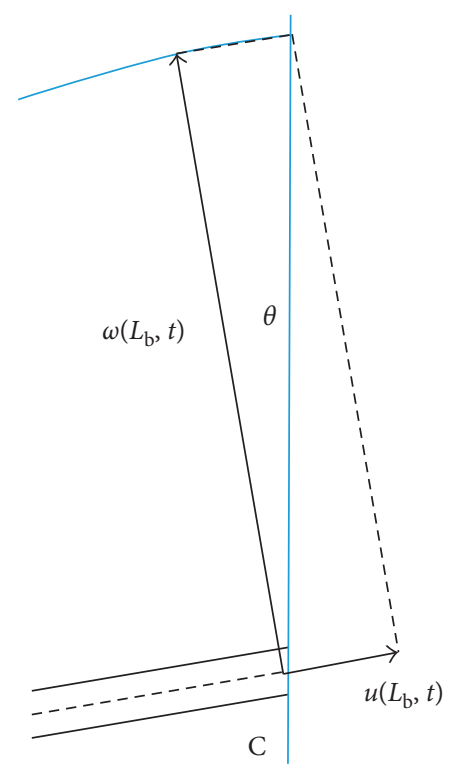

(a)

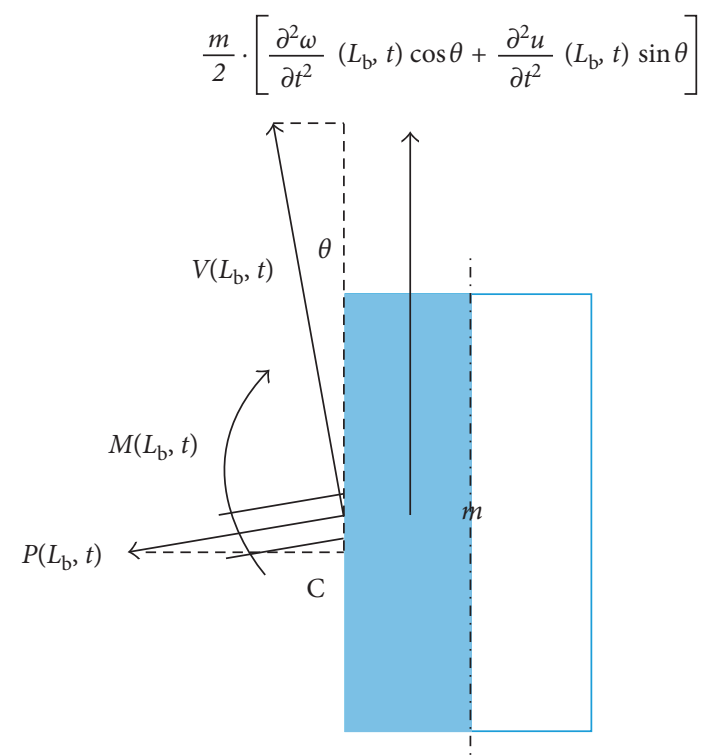

(b)

FIgURE 3: Continuity conditions.

TABle 2: Material properties and dimensions (unit of length: $\mu \mathrm{m}$ ).

\begin{tabular}{lccccccc}
\hline$\rho\left(\mathrm{kg} \cdot \mathrm{m}^{-3}\right)$ & $E\left(\mathrm{~N} \cdot \mathrm{m}^{-2}\right)$ & $b$ & $h_{0}$ & $h_{1}$ & $L_{0}$ & $L_{2}$ & $L_{\mathrm{s}}$ \\
\hline 2330 & $160 \times 10^{9}$ & 20 & 4 & 100 & 250 & 30 & 60
\end{tabular}

(known as the angular frequency) is called the natural frequency. In this section, $f_{n}\left(=\left(\omega_{n} / 2 \pi\right)\right)$ is referred to as the natural frequency in unit $\mathrm{Hz}$. Since the beam is of the continuous system with the infinite number of degrees of freedom, infinite orders of natural frequencies and associated mode shapes can be obtained. In this section, only the first three orders of natural frequencies and mode shapes are computed and compared, as summarized in Table 3. The percent deviations are calculated as follows: $\Delta \%=$ $\left(f_{\text {analytical }}-f_{\text {ANSYS }} / f_{\text {ANSYS }}\right) \times 100$.

It can be seen from Table 3 that the analytical results agree well with the simulation results with a maximum derivation of $1.11 \%$ for predicting the natural frequencies of the $\mathrm{V}$-shaped beams with a shuttle, and a maximum derivation of $-0.44 \%$ for the $\mathrm{V}$-shaped beam without a shuttle. The analytically calculated mode shapes are based on the combination of the calculations of the lateral and longitudinal mode shapes, as in (20) and (21). The mode shapes calculated analytically also match the FEA simulation results very well. Clearly, the natural frequencies for the case without a shuttle are higher than the results for the case with a shuttle. This can be explained in such way that increasing the mass results in decreasing the natural frequencies for such a spring-mass system by considering the pair of beam to be a spring carrying a mass. In this comparison, the asymmetric and out-of-plane vibrations are not taken into consideration since these cases are irrelevant to the direction of actuation. For the multipairs of beam case, since each pairs of beams are identical and symmetrically configured, the mode shapes for each pairs are the same and the natural frequencies can be calculated based on the single pair of beam with the mass of the shuttle being evenly distributed for each pairs of beams.

\section{Volume Scaling}

For MEMS machining process, electrothermal microactuators can be fabricated with the beam length ranging from hundreds to thousands of microns and even larger. One of the problems that has to be addressed at the early stage during the design process is to determine the volume scale of the $\mathrm{V}$-shaped electrothermal microactuators. In general, in order to achieve larger displacement and force, it is desirable if we can just simply scale up the volume. However, this will decrease the electrothermal response, and it may not be suited to fast actuation applications. On the contrary, scaling up the volume will result in decreasing of the natural frequencies and the impact of the mechanical vibrations can increase.

In this section, we investigate the relationship between natural frequencies and volume scale. First, we introduce the scale factor $S_{f}$, and the beam dimensions can be written with respect to the reference dimensions as $L_{\mathrm{b}}=L_{\mathrm{b}}^{*} S_{\mathrm{f}}, L_{0}=L_{0}^{*} S_{\mathrm{f}}, \quad L_{2}=L_{2}^{*} S_{\mathrm{f}}, \quad b=b^{*} S_{\mathrm{f}}, \quad h_{0}=h_{0}^{*} S_{\mathrm{f}}$, $h_{1}=h_{1}^{*} S_{\mathrm{f}}$, and $L_{\mathrm{s}}=L_{\mathrm{s}}^{*} S_{\mathrm{f}}$, where $L_{\mathrm{b}}^{*}, L_{0}^{*}, L_{2}^{*}, b^{*}, h_{0}^{*}$, and $h_{1}^{*}$ are called the reference beam dimensions. The cross-sectional area, moment of inertia, and mass of the shuttle of $\mathrm{V}$-shaped beam corresponding to the reference beam dimensions are calculated as $A^{*}=b^{*} h_{0}^{*}, I^{*}=\left(b^{*} h_{0}^{* 3} / 12\right)$, and $m^{*}=\rho b^{*} h_{1}^{*} L_{\mathrm{s}}^{*}$, respectively. For a particular $\mathrm{V}$-shaped beam with a factor $S_{\mathrm{f}}$, we have $A=A^{*} S_{\mathrm{f}}^{2}, I=I^{*} S_{\mathrm{f}}^{4}$, and $m=m^{*} S_{\mathrm{f}}^{3}$.

Note that the inclined angle remains to be the same with the beam scaling up or down as $\theta=a \tan \left(L_{2} / L_{0}\right)=$ $a \tan \left(L_{2}^{*} / L_{0}^{*}\right)$. With this, (8) and (9) can be rewritten as 
TABLE 3: Comparison between analytical and simulation results.

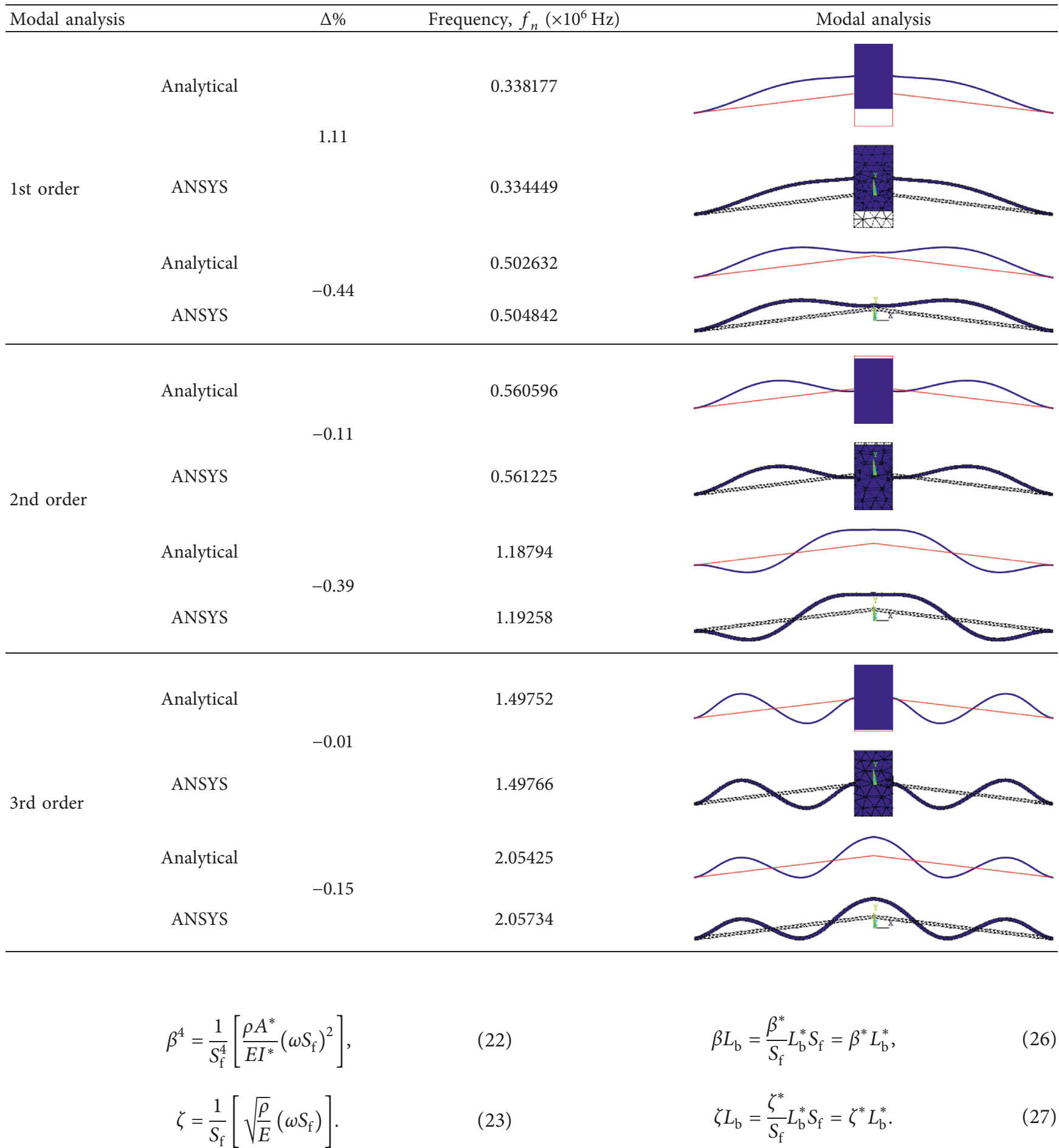

Write

$$
\begin{aligned}
& \beta=\frac{\beta^{*}}{S_{\mathrm{f}}}, \\
& \zeta=\zeta^{*} \frac{1}{S_{\mathrm{f}}},
\end{aligned}
$$

where $\beta^{*}=\left[\left(\rho A^{*} / \mathrm{EI}^{*}\right)\left(\omega \mathrm{S}_{\mathrm{f}}\right)^{2}\right]^{(1 / 4)}$ and $\zeta^{*}=\sqrt{(\rho / E)}\left(\omega S_{\mathrm{f}}\right)$. We have

Denoting $\quad s^{*}=\sin \left(\beta^{*} L_{\mathrm{b}}^{*}\right), \quad c^{*}=\cos \left(\beta^{*} L_{\mathrm{b}}^{*}\right), \quad s h^{*}=$ $\sinh \left(\beta^{*} L_{\mathrm{b}}^{*}\right), c h^{*}=\cosh \left(\beta^{*} L_{\mathrm{b}}^{*}\right), \bar{s}^{*}=\sin \left(\zeta^{*} L_{\mathrm{b}}^{*}\right)$, and $\bar{c}^{*}=$ $\cos \left(\zeta^{*} L_{\mathrm{b}}^{*}\right)$, we have $s=s^{*}, c=c^{*}, s h=s h^{*}, c h=c h^{*}, \bar{s}=\bar{s}^{*}$, and $\bar{c}=\bar{c}^{*}$. Clearly, since $\beta^{*}$ and $\zeta^{*}$ are the functions of $\omega S_{\mathrm{f}}$, $s^{*}, c^{*}, s h^{*}, c h^{*}, \bar{s}^{*}$, and $\bar{c}^{*}$ are the functions of $\omega S_{\mathrm{f}}$. The last row in (13) is expressed as

$$
a_{31} C_{2}+a_{32} C_{4}+a_{33} D_{2}=0 \text {. }
$$

Considering (24) and (25), $a_{31}, a_{32}$, and $a_{33}$ can be rewritten as 


$$
\begin{aligned}
& a_{31}=S_{\mathrm{f}} a_{31}^{*}, \\
& a_{32}=S_{\mathrm{f}} a_{32}^{*}, \\
& a_{33}=S_{\mathrm{f}} a_{33}^{*},
\end{aligned}
$$

where $a_{31}^{*}=\left[2 E I^{*}\left(s^{*}-s h^{*}\right) \beta^{* 3}+m^{*}\left(\omega S_{\mathrm{f}}\right)^{2}\left(c^{*}-c h^{*}\right)\right] \cos \theta$, $a_{32}^{*}=\left[2 E I^{*}\left(-c^{*}-c h^{*}\right) \beta^{* 3}+m^{*}\left(\omega S_{\mathrm{f}}\right)^{2}\left(s^{*}-s h^{*}\right)\right] \cos \theta$, and $a_{33}^{*}=\left(-2 E A^{*} \zeta^{*} \bar{c}^{*}+m^{*}\left(\omega S_{\mathrm{f}}\right)^{2} \bar{s}^{*}\right) \sin \theta$. Introducing (29), (30), and (31) into (28) leads to

$$
a_{31}^{*} C_{2}+a_{32}^{*} C_{4}+a_{33}^{*} D_{2}=0 .
$$

Considering (32), (13) can be written with respect to the reference beam dimensions, and all the elements in the coefficient matrix are the functions of $\omega S_{\mathrm{f}}$. Therefore, the determinant of the coefficient matrix must be a function of $\omega S_{\mathrm{f}}$, which corresponds to the reference beam dimensions. That is, the frequency equations (14) and (15) are both functions of $\omega S_{f}$ :

$$
\begin{aligned}
& m^{*}\left(\omega S_{\mathrm{f}}\right)^{2} \bar{s}^{*}\left(1-c^{*} \cdot c h^{*}\right)= 2 \zeta^{*} E A^{*} \bar{c}^{*}\left(1-c^{*} \cdot c h^{*}\right) \sin ^{2} \theta \\
&+2 \beta^{* 3} E I^{*} \bar{s}^{*} \\
& \cdot\left(s^{*} \cdot c h^{*}+c^{*} \cdot s h^{*}\right) \cos ^{2} \theta \\
& \zeta^{*} A^{*} \bar{c}^{*}\left(1-c^{*} \cdot c h^{*}\right) \sin ^{2} \theta \\
&+\beta^{* 3} I^{*} \bar{s}^{*}\left(s^{*} \cdot c h^{*}\right. \\
&\left.+c^{*} \cdot s h^{*}\right) \cos ^{2} \theta=0 .
\end{aligned}
$$

Comparing (14) and (15) with (33) and (34), we can conclude that $\omega S_{\mathrm{f}}$ is the natural frequency corresponding to the reference beam dimensions, that is, $\omega^{*}=\omega S_{\mathrm{f}}$. Hence, we have

$$
\omega_{n}=\frac{\omega_{n}^{*}}{S_{\mathrm{f}}}
$$

where the subscript $n$ indicate the orders of the natural frequencies. Equation (35) is important for predicting the natural frequencies of the $\mathrm{V}$-shaped beam actuator. If the natural frequencies of one particular $\mathrm{V}$-shaped actuator are given, the natural frequencies for the scaled actuator with the scale factor $S_{\mathrm{f}}$ can also be obtained. Clearly, the natural frequencies are inversely proportional to the volume scale for the same dimension proportions and same material properties.

This relationship is demonstrated in Figure 4, (35). Figures 4(a) and 4(b) are based upon the V-shaped beam dimensions listed in Table 2, which correspond to the scale factor $S_{\mathrm{f}}=1$. The material properties used in Figures 4(a) and 4(b) are silicon (listed in Table 2) and SU-8, respectively. Young's modulus and density of SU-8 are $2.1 \times 10^{9}\left(\mathrm{~N} \cdot \mathrm{m}^{-2}\right)$ and $2150\left(\mathrm{~kg} \cdot \mathrm{m}^{-3}\right)$. It is seen that the calculated natural frequencies are indeed inversely proportional to the volume scale $S_{\mathrm{f}}$. This suggests that when the $\mathrm{V}$-shaped beam is scaled up, the impact of the mechanical vibration on the dynamics of the V-shaped beam increases. Compared to silicon, the natural frequencies of the $\mathrm{V}$-shaped beam made of SU-8 is nearly 10 times smaller. This indicates that the material properties also have a large impact on the vibrations of the $\mathrm{V}$-shaped beam microactuators. In what follows, we will discuss in detail the influence of the material and dimensions of the structure on the natural frequencies.

\section{Parameter Analysis}

In this section, we investigate the influence of material properties and beam dimensions on the natural frequencies in order to provide insight and guidance on the further design of the $\mathrm{V}$-shaped beam electrothermal microactuators.

5.1. Material Properties. It is seen from the frequency equation that only Young's modulus $E$ and density $\rho$ of the actuator determine the natural frequencies if beam dimensions are fixed. $\sqrt{(\rho / E)}$ may be called the characteristics material property for vibration. For a reference characteristics material property $\sqrt{(\tilde{\rho} / \widetilde{E})}, \sqrt{(\rho / E)}$ relates to $\sqrt{(\tilde{\rho} / \widetilde{E})}$ with a scale factor $S_{\mathrm{f}}^{m}$ as follows:

$$
\sqrt{\frac{\rho}{E}}=\sqrt{\frac{\widetilde{\rho}}{\widetilde{E}}} \cdot S_{\mathrm{f}}^{m}
$$

Introducing (36) into (8) and (9) leads to

$$
\begin{aligned}
& \beta=\left[\frac{\tilde{\rho}}{\widetilde{E}} \frac{A}{I}\left(\omega S_{\mathrm{f}}^{m}\right)^{2}\right]^{(1 / 4)}, \\
& \zeta=\sqrt{\frac{\widetilde{\rho}}{\widetilde{E}}} \cdot\left(\omega S_{\mathrm{f}}^{m}\right), \\
& \tilde{\beta}=\left[\frac{\tilde{\rho}}{\widetilde{E}} \frac{A}{I}\left(\omega S_{\mathrm{f}}^{m}\right)^{2}\right]^{(1 / 4)}, \\
& \tilde{\zeta}=\sqrt{\frac{\widetilde{\rho}}{\widetilde{E}}} \cdot\left(\omega S_{\mathrm{f}}^{m}\right),
\end{aligned}
$$

and comparing (39) and (40) with (8) and (9), we notice that $\beta$ and $\zeta$ are the functions of $\omega$ for characteristics material properties $\sqrt{(\rho / E)}$, whereas $\widetilde{\beta}$ and $\widetilde{\zeta}$ are the functions of $\omega S_{\mathrm{f}}^{m}$ for the reference characteristics material properties $\sqrt{(\widetilde{\rho} / \widetilde{E})}$. Obviously, we have

$$
\begin{aligned}
& \beta=\tilde{\beta}, \\
& \zeta=\widetilde{\zeta}
\end{aligned}
$$

Denoting $\widetilde{s}=\sin \left(\widetilde{\beta} L_{\mathrm{b}}\right), \quad \widetilde{c}=\cos \left(\widetilde{\beta} L_{\mathrm{b}}\right), \widetilde{s h}=\sinh \left(\widetilde{\beta} L_{\mathrm{b}}\right)$, $\tilde{c h}=\cosh \left(\widetilde{\beta} L_{\mathrm{b}}\right), \tilde{\bar{s}}=\sin \left(\widetilde{\zeta} L_{\mathrm{b}}\right)$, and $\tilde{\bar{c}}=\cos \left(\widetilde{\zeta} L_{\mathrm{b}}\right)$, we have $s=\widetilde{s}, c=\widetilde{c}, s h=\widetilde{s h}, c h=\widetilde{c h}, \bar{s}=\widetilde{\bar{s}}$, and $\bar{c}=\widetilde{\bar{c}}$. The mass of the shuttle $m$ is calculated as $m=\rho V_{s}$, in which $V_{s}$ is the volume of the shuttle. For the reference density $\tilde{\rho}$, mass of the shuttle is calculated as $\tilde{m}=\tilde{\rho} V_{s}$. Clearly,

$$
\frac{m \omega^{2}}{E}=\frac{\rho V_{\mathrm{s}} \omega^{2}}{E}=\frac{\widetilde{\rho} V_{\mathrm{s}}\left(\omega S_{\mathrm{f}}^{m}\right)^{2}}{\widetilde{E}}=\frac{\widetilde{m}\left(\omega S_{\mathrm{f}}^{m}\right)^{2}}{\widetilde{E}} .
$$

Applying (43) into (28), $a_{31}, a_{32}$, and $a_{33}$ can be expressed as 


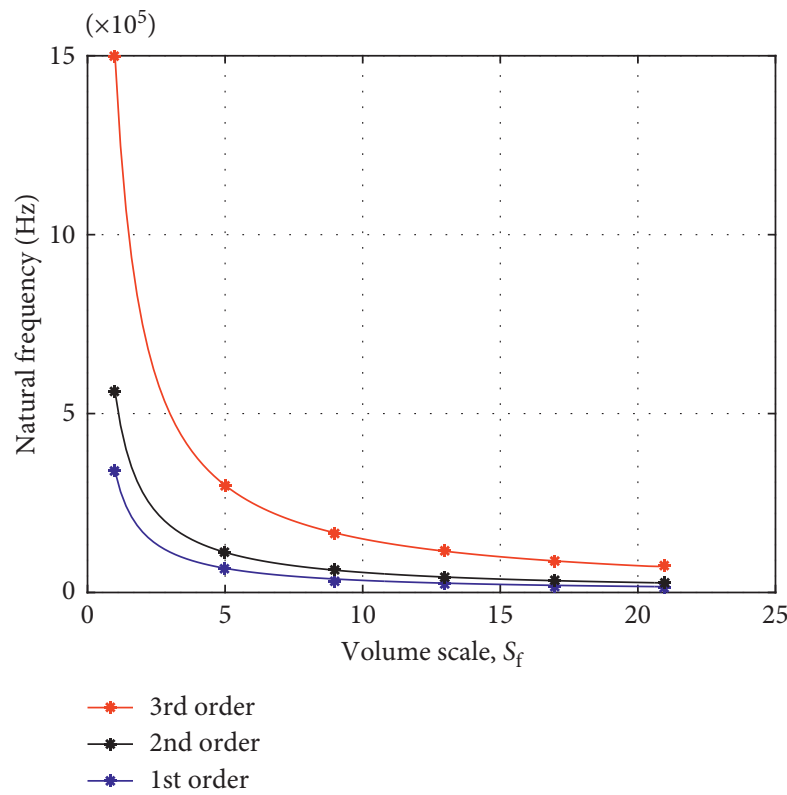

(a)

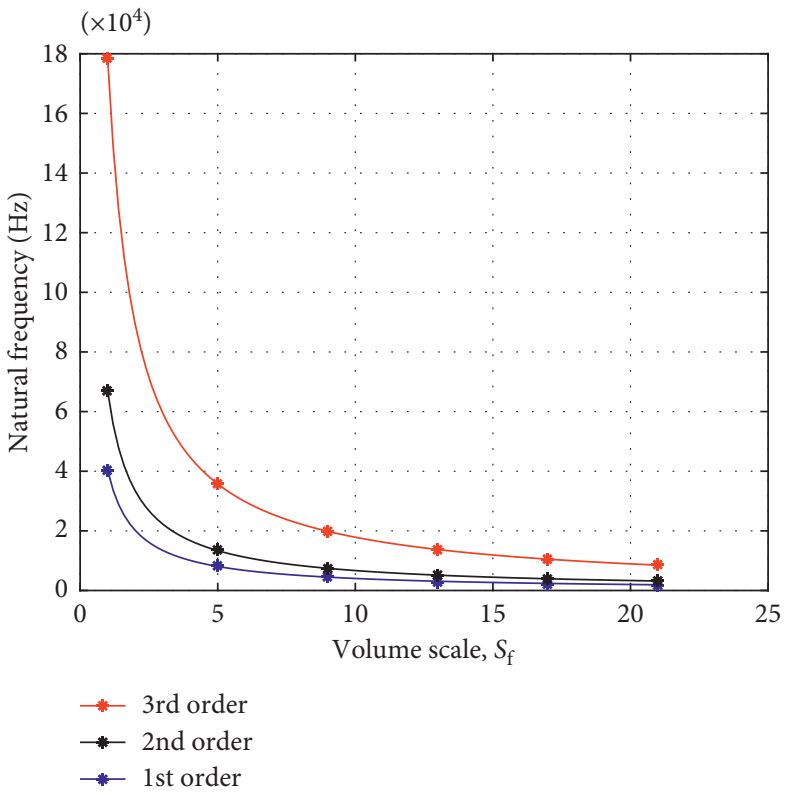

(b)

FIgURE 4: Natural frequencies versus volume scale. (a) Silicon. (b) SU-8.

$$
\begin{aligned}
& a_{31}=\frac{E}{\widetilde{E}} \widetilde{a}_{31}, \\
& a_{32}=\frac{E}{\widetilde{E}} \widetilde{a}_{32}, \\
& a_{33}=\frac{E}{\widetilde{E}} \widetilde{a}_{33},
\end{aligned}
$$

where $\tilde{a}_{31}=\left[2 \widetilde{E} I(\widetilde{s}-\widetilde{s h}) \widetilde{\beta}^{3}+\widetilde{m}\left(\omega S_{f}^{m}\right)^{2}(\widetilde{c}-\widetilde{c h})\right] \cos \theta, \quad \tilde{a}_{32}=$ $\left[2 \widetilde{E} I(-\widetilde{c}-\widetilde{c h}) \widetilde{\beta}^{3}+\widetilde{m}\left(\omega S_{\mathrm{f}}^{m}\right)^{2}(\widetilde{s}-\widetilde{s})\right] \cos \theta$, and $\widetilde{a}_{33}=[-2 \widetilde{E} A \widetilde{\zeta} \bar{c}+$ $\left.\widetilde{m}\left(\omega S_{f}^{m}\right)^{2} \widetilde{\bar{s}}\right] \sin \theta$. Introducing (44), (45), and (46) into (28) leads to

$$
\widetilde{a}_{31} C_{2}+\widetilde{a}_{32} C_{4}+\widetilde{a}_{33} D_{2}=0 .
$$

Considering (47), all the elements in the coefficient matrix in (13) are the functions of $\omega S_{\mathrm{f}}^{m}$, and therefore, the determinant of the coefficient matrix, that is, the frequency equation, is the function of $\omega S_{\mathrm{f}}^{m}$. The frequency (14) and (15) are expressed as

$$
\begin{aligned}
\widetilde{m}\left(\omega S_{\mathrm{f}}^{m}\right)^{2} \widetilde{\bar{s}}(1-\widetilde{c} \cdot \widetilde{c h})= & 2 \widetilde{\zeta} \widetilde{E} A \widetilde{\bar{c}}(1-\widetilde{c} \cdot \widetilde{c h}) \sin ^{2} \theta \\
& +2 \widetilde{\beta}^{3} \widetilde{E} I \widetilde{\bar{s}}(\widetilde{s} \cdot \widetilde{c h}+\widetilde{c} \cdot \widetilde{s h}) \cos ^{2} \theta,
\end{aligned}
$$

$$
\begin{aligned}
\widetilde{\zeta} A \widetilde{\bar{c}}(1-\tilde{c} \cdot \tilde{c h}) \sin ^{2} \theta \\
\quad+\widetilde{\beta}^{3} I \tilde{\bar{s}}(\widetilde{s} \cdot \tilde{c h} \\
\quad+\widetilde{c} \cdot \widetilde{s h}) \cos ^{2} \theta=0 .
\end{aligned}
$$

Comparing (48) and (49) with (14) and (15), we can conclude that $\omega S_{\mathrm{f}}^{m}$ represents the natural frequencies corresponding to the reference characteristics material properties, that is, $\widetilde{\omega}=\omega S_{\mathrm{f}}^{m}$. Hence, we have

$$
\omega_{n}=\frac{\widetilde{\omega}_{n}}{S_{\mathrm{f}}^{m}}
$$

where the subscript $n$ indicate the orders of the natural frequencies. Equation (50) demonstrates that the natural frequencies are inversely proportional to the scale factor $S_{\mathrm{f}}^{m}$. Combining (35) and (50) leads to

$$
\omega_{n}=\frac{\widetilde{\omega}_{n}^{*}}{S_{\mathrm{f}} \cdot S_{\mathrm{f}}^{m}},
$$

where $\widetilde{\omega}_{n}^{*}$ represents the natural frequencies associated to the reference beam dimensions and characteristics material properties. Though $S_{\mathrm{f}}^{m}$ and $S_{\mathrm{f}}$ are both called the scale factor, they are different. $S_{\mathrm{f}}$ refers to the scaling of the volume of the actuator, and $S_{\mathrm{f}}^{m}$ refers to scaling the value of the characteristics material property $\sqrt{(\rho / E)}$.

Table 4 lists six materials that are commonly used for fabrication of electrothermal microactuators. The characteristics material property of the polysilicon is the smallest and was considered as the reference material. The materials relate the polysilicon with the scale factor $S_{\mathrm{f}}^{m}$ from 1.00 to 8.70 according to the relative value of the characteristics material properties $\sqrt{(\rho / E)}$.

Figure 5 shows the natural frequencies with respect to the scale factor $S_{f}^{m}$, in which the solid line represents the natural frequencies derived from (50) with the natural frequencies of polysilicon as the reference value. The asterisks represent the analytically calculated natural frequencies of particular material properties (as listed in Table 4) based upon the modal analysis in Section 2. The beam dimensions are chosen as in Table 2. Clearly, the asterisks fall exactly on the solid lines as predicted by (50). For the same beam dimensions, the natural frequencies of 
TABLE 4: Material properties comparisons.

\begin{tabular}{lcccccc}
\hline & Polysilicon [43] & Silicon [44] & Gold [43] & Aluminium [43] & Nickel [43] & SU-8 [45] \\
\hline$S_{\mathrm{f}}^{m}$ & 1.00 & 1.04 & 1.35 & 1.56 & 1.77 & 8.70 \\
$\sqrt{(\rho / E)}$ & 3.68 & 3.82 & 4.97 & 5.73 & 6.52 & 32.00 \\
$\rho\left(\mathrm{kg} \cdot \mathrm{m}^{-3}\right)$ & 2230 & 2330 & 1930 & 2300 & 8920 \\
$E(\mathrm{GPa})$ & 165 & 160 & 78 & 70 & 210 & 2.1 \\
\hline
\end{tabular}

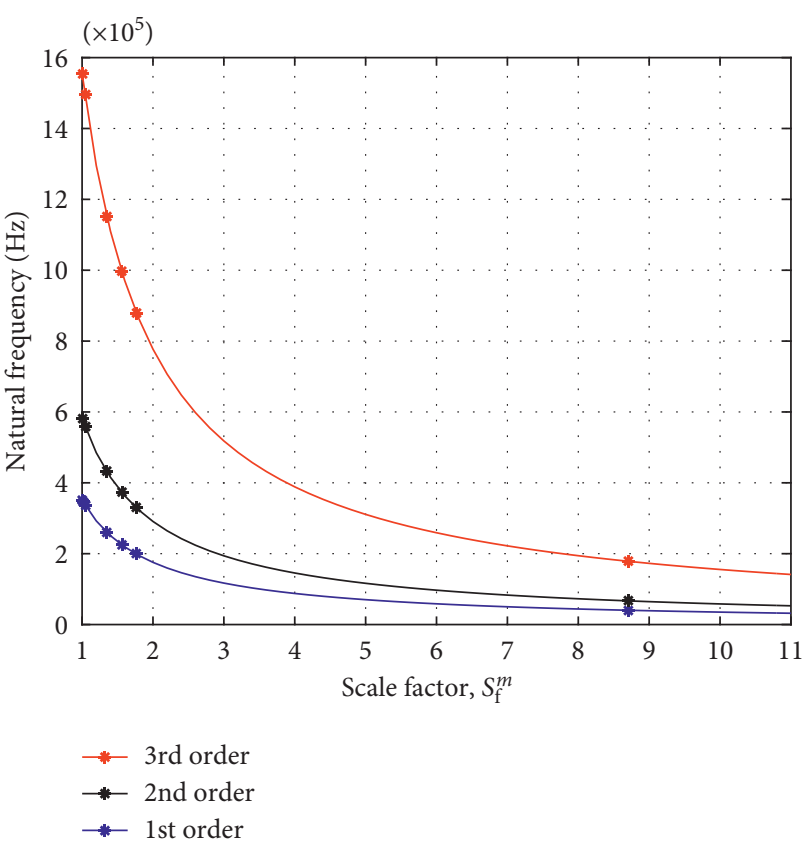

Figure 5: Natural frequencies versus scale factor $S_{\mathrm{f}}^{m}$ : from left to right the asterisk represents polysilicon, silicon, gold, aluminum, nickel, and SU-8, respectively.

$\mathrm{V}$-shaped beam made of polysilicon are the largest, meaning that, in this case, the dominant dynamics is determined by the electrothermal part and the influence of the mechanical vibration is small and negligible. The $\mathrm{V}$-shaped beam made of SU-8 is most likely to undergo mechanical vibrations since the natural frequencies are the smallest.

5.2. Structural Parameters. In this section, the effect of varying the structural parameters, that is, $L_{2}, L_{0}$, and $h_{0}$, of the $\mathrm{V}$-shaped beam on its natural frequencies is studied. The thickness of the $\mathrm{V}$-shaped beam $b$ is not studied since it is irrelevant to the natural frequencies as demonstrated in (14) and (15). In the following discussions, we consider a single pair of beam with a shuttle for illustration, and similar conclusions can be extended to the cases of multipairs of beam and beam without a shuttle.

The effect of changing $L_{2}$ on the first-order natural frequencies is shown in Figure 6. The natural frequency increases to the maximum value followed by a drop as $L_{2}$ increases. In the meantime, the mode shape also changes with increasing $L_{2}$, as shown in Figure 7. Obviously, the motion of the shuttle contributes to the vibration mode for $L_{2}$ less than around $60 \mu \mathrm{m}$. As $L_{2}$ increases, the vibration mode of the structure tends not to involve the motion of the

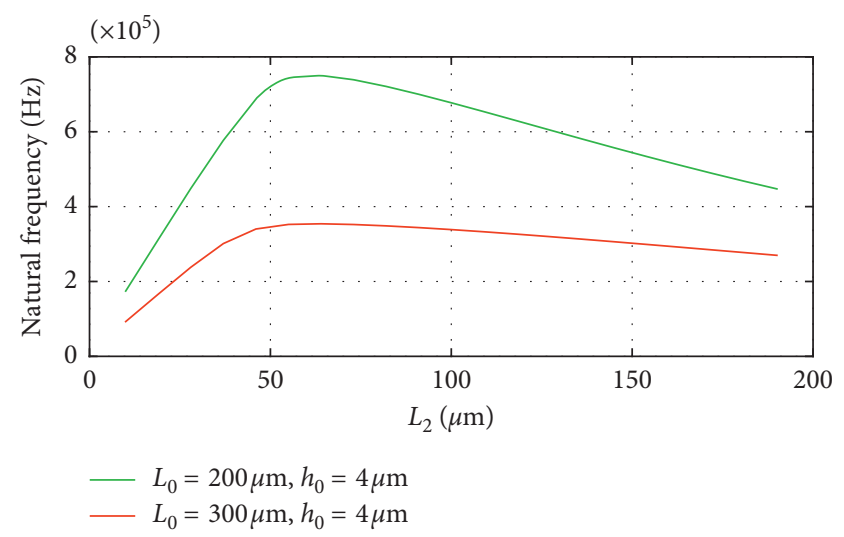

FIgURE 6: Natural frequency versus $L_{2}$.

shuttle. For the first case, as shown in Figure 7(a), the $\mathrm{V}$-shaped beam can be thought of as a spring-mass system in which the mass of the shuttle represents the equivalent mass of the system and the stiffness of that system is the stiffness in the direction of the motion of the shuttle. Apparently, increasing $L_{2}$ leads to larger longitudinal deflections thus increasing the stiffness of the system. For a spring-mass system, increasing the stiffness will lead to increase in the natural frequencies. For the latter case, as shown in Figure 7(b), the V-shaped beam can be thought of as a fixedfixed line-shaped beam for the half of the structure. With an increase of $L_{2}$, the stiffness for this fixed-fixed beam decreases resulting in decreasing of the natural frequencies. Clearly, Figures 6 and 7 demonstrate that varying $L_{2}$ will not only influence the natural frequency but also influence the mode shape.

The effect of varying $L_{0}$ on the first-order natural frequency is illustrated in Figure 8. The green and the red curves represent the two classes of vibration modes, that is, the spring-mass and the fixed-fixed cases, respectively. For both vibration modes, as shown in Figure 8, the first-order natural frequency decreases with increasing $L_{0}$. Obviously, increasing $L_{0}$ results in the decrease of the stiffness of the structure. Figure 9 shows the first-order natural frequencies with respect to beam width $h_{0}$. The red and green curves represent the two vibration modes. Clearly, for both the modes, increasing the beam width results in stiffening the beam thus increasing the natural frequencies.

\section{Conclusions}

A comprehensive analytical modal analysis and a parameter study of V-shaped beam electrothermal microactuators have been conducted for the first time in this work. Both the 

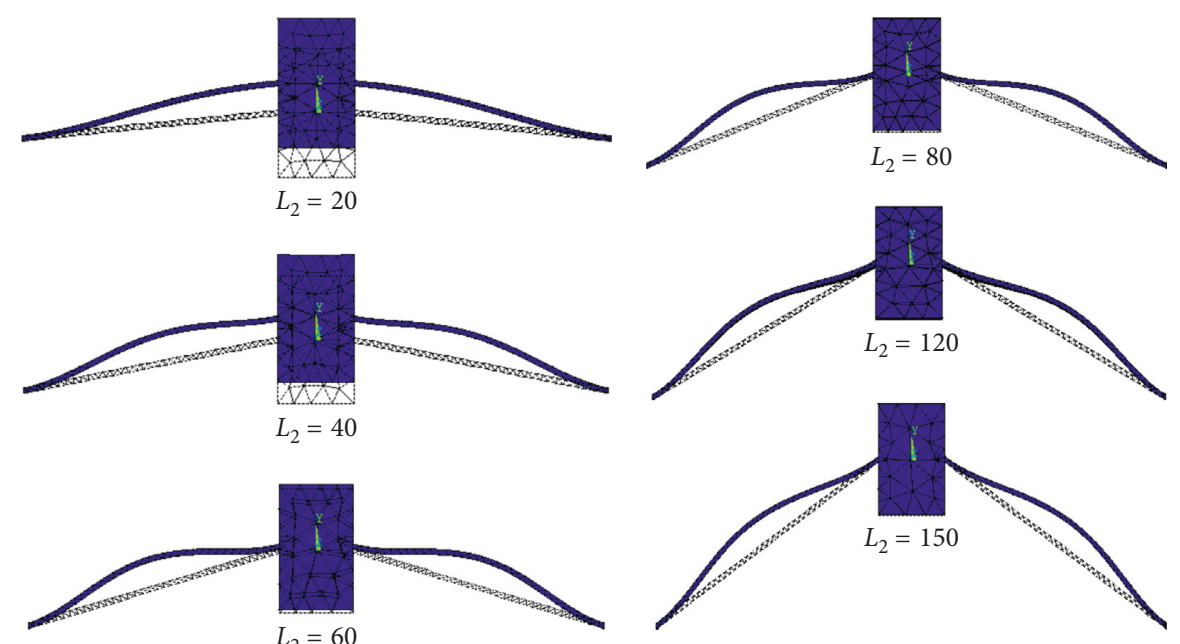

(a)

(b)

Figure 7: Mode shapes for $L_{0}=200 \mu \mathrm{m}$.

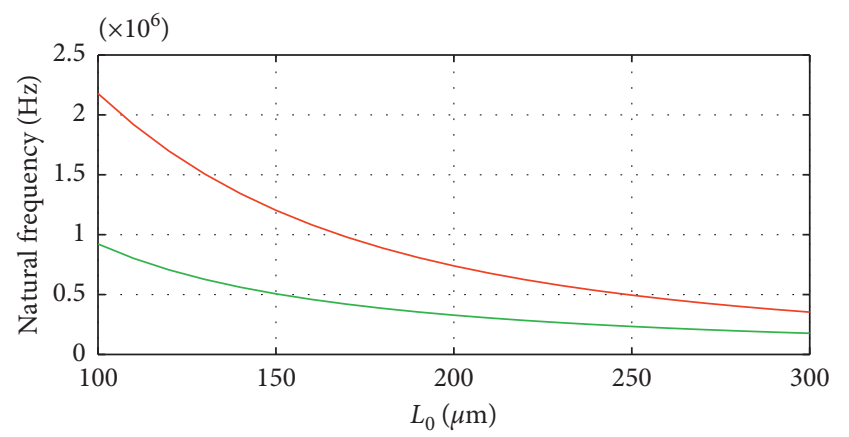

- $L_{2}=20 \mu \mathrm{m}, h_{0}=4 \mu \mathrm{m}$

- $L_{2}=70 \mu \mathrm{m}, h_{0}=4 \mu \mathrm{m}$

Figure 8: Natural frequency versus $L_{0}$.

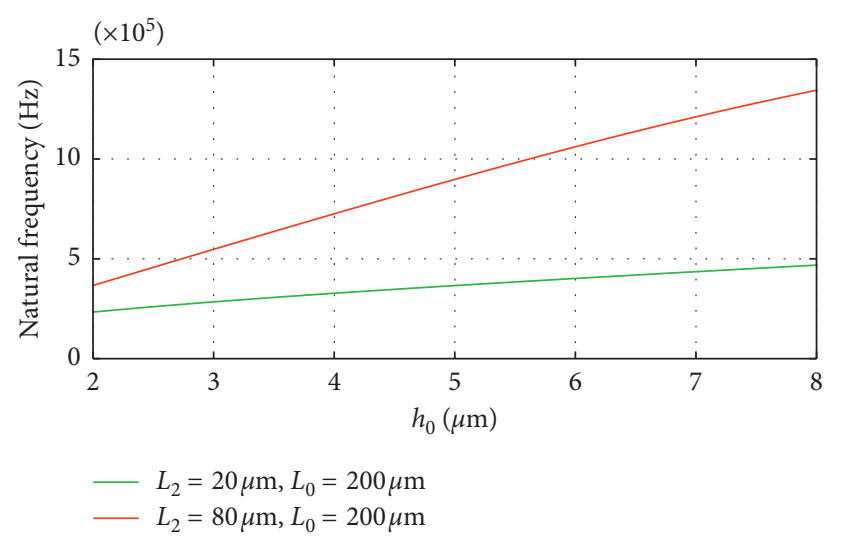

FIGURE 9: Natural frequency versus $h_{0}$.

longitudinal and lateral vibrations have been included in deriving the natural frequencies and the corresponding mode shapes of the V-shaped beam. Analytically obtained natural frequencies and mode shapes were compared to the results of finite element simulations using ANSYS to verify the analytical modal analysis. In addition, this paper has analytically proved that the natural frequencies are inversely proportional to the volume scale and the scale factor of characteristic material properties. This provides the guidance on how to determine the volume scale and to choose the materials of the V-shaped beam electrothermal microactuators. Finally, the parameter analysis was conducted to provide insights on the design and optimization of the V-shaped electrothermal microactuators.

\section{Conflicts of Interest}

The authors declare that there are no conflicts of interest regarding the publication of this paper.

\section{Acknowledgments}

This work was supported by the National Natural Science Foundation of China (no. 51575006).

\section{References}

[1] X. Li, L. Lang, J. Liu et al., "Electro-thermally actuated RF MEMS switch for wireless communication," in Proceedings of 2010 5th International Conference on Nano/Micro Engineered and Molecular Systems, pp. 497-500, Xiamen, China, 2010.

[2] S. Karajgikar, S. Rao, J. Sin et al., "Electro-thermal analysis of in-plane micropump," IEEE Transactions on Components and Packaging Technologies, vol. 33, no. 2, pp. 329-339, 2010.

[3] P. Shivhare, G. Uma, and M. Umapathy, "Design enhancement of a chevron electrothermally actuated microgripper for improved gripping performance," Microsystem Technologies, vol. 22, no. 11, pp. 2623-2631, 2015.

[4] Z. Wang, X. Shen, and X. Chen, "Design, modeling, and characterization of a MEMS electrothermal microgripper," Microsystem Technologies, vol. 21, no. 11, pp. 2307-2314, 2015.

[5] M. Rakotondrabe, A. G. Fowler, and S. O. Reza, "Control of a novel 2-DoF MEMS nanopositioner with electrothermal 
actuation sensing," IEEE Transactions on Control Systems Technology, vol. 22, no. 4, pp. 1486-1497, 2014.

[6] S. Oak, S. Rawool, G. Sivakumar et al., "Development and testing of a multilevel chevron actuator-based positioning system," Journal of Microelectromechanical Systems, vol. 20, no. 6, pp. 1298-1309, 2011.

[7] Y. Zhu, A. Corigliano, and H. D. Espinosa, "A thermal actuator for nanoscale in situ microscopy testing: design and characterization," Journal of Micromechanics and Microengineering, vol. 16, no. 2, pp. 242-253, 2006.

[8] S. Gupta, T. Pahwa, R. Narwal, B. Prasad, and D. Kumar, "Optimizing the performance of MEMS electrostatic comb drive actuator with different flexure springs," in Proceedings of 2012 COMSOL Conference, Bangalore, India, 2012.

[9] R. E. Pawinanto, J. Yunas, B. Y. Majlis, and A. A. Hamzah, "Finite element analysis on magnetic force generation of electromagnetic microactuator for micropump," in Proceedings of 2013 IEEE Regional Symposium on Micro and Nanoelectronics, pp. 25-28, Langkawi, Malaysia, September 2013.

[10] R. E. K. Moussa, M. Grossard, M. Boukallel, A. Hubert, and N. Chaillet, "Modeling and control of a piezoelectric microactuator with proprioceptive sensing capabilities," Measurement, vol. 24, no. 6, pp. 590-604, 2014.

[11] D. Sameoto, T. Hubbard, and M. Kujath, "Operation of electrothermal and electrostatic MUMPs microactuators underwater," Journal of Micromechanics and Microengineering, vol. 14, no. 10, pp. 1359-1366, 2014.

[12] M. Mayyas, P. S. Shiakolas, W. H. Lee, and H. Stephanou, "Thermal cycle modeling of electrothermal microactuators," Sensor Actuator A: Physical, vol. 152, no. 2, pp. 192-202, 2009.

[13] R. Venditti, J. S. H. Lee, Y. Sun, and D. Li, "An in-plane, bidirectional electrothermal MEMS actuator," Journal of Micromechanics and Microengineering, vol. 16, no. 10, pp. 2067-2070, 2006.

[14] L. Li and D. Uttamchandani, "Dynamic response modelling and characterization of a vertical electrothermal actuator," Journal of Micromechanics and Microengineering, vol. 19, no. 7, article 075014, 2009.

[15] K. Ogando, N. Forgia, J. Zarate, and H. Pastoriza, "Design and characterization of a fully compliant out-of-plane thermal actuator," Sensor Actuator A: Physical, vol. 183, pp. 95-100, 2012.

[16] C. Elbuken, N. Topaloglu, P. Nieva, M. Yavuz, and J. Huissoon, "Modeling and analysis of a 2-DOF bidirectional electro-thermal microactuator," Microsystem Technologies, vol. 15, no. 5, pp. 713-722, 2009.

[17] X. Chen and D. Lee, "A microcantilever system with slidercrank actuation mechanism," Sensors and Actuators A: Physical, vol. 226, pp. 59-68, 2015.

[18] M. Kaur and S. Dhariwal, "Parameters affecting electrothermal actuation based microtweezers," International Journal of Current Engineering and Technology, vol. 2, no. 3, pp. 305-309, 2012.

[19] B. Jyoti and S. Halse, "Modelling and simulation of three arm electrothermal actuator using Comsol," International Journal on Emerging Technologies, vol. 7, no. 2, pp. 261-264, 2016.

[20] R. K. Messenger, Q. T. Aten, T. W. McLain, and L. L. Howell, "Piezoresistive feedback control of a MEMS thermal actuator," Journal of Microelectromechanical Systems, vol. 18, no. 6, pp. 1267-1278, 2007.

[21] C. Lee, "A MEMS VOA using electrothermal actuators," Journal of Lightwave Technology, vol. 25, no. 2, pp. 490-498, 2007.
[22] C. Guan and Y. Zhu, "An electrothermal microactuator with Z-shaped beams," Journal of Micromechanics and Microengineering, vol. 20, no. 8, article 085041, 2010.

[23] Z. Zhang, W. Zhang, Q. Wu, Y. Yu, X. Liu, and X. Zhang, "A comprehensive analytical model and experimental validation of Z-shaped electrothermal microactuators," in Mechanisms and Machine Science, pp. 177-187, Springer, Berlin, Germany, 2015.

[24] Z. Zhang, Y. Yu, and X. Zhang, "Theoretical modal analysis and parameter study of Z-shaped electrothermal microactuators," Microsystem Technologies, vol. 24, no. 7, pp. 3149-3160, 2018.

[25] V. Vidyaa and G. Arumaikkannu, "Hybrid design of a polymeric electrothermal actuator for microgripper," International Journal of Mechanical and Industrial Engineering, vol. 1, no. 2, pp. 2231-6477, 2011.

[26] D. Mallick, P. K. Podder, and A. Bhattacharyya, "Design and simulation of MEMS based thermally actuated positioning systems," in Proceedings of 2012 5th International Conference on Computers and Devices for Communication (CODEC), pp. 1-4, Kolkata, India, December 2012.

[27] Z. Zhang, Y. Yu, X. Liu, and X. Zhang, "Dynamic modelling and analysis of $\mathrm{V}$ - and Z-shaped electrothermal microactuators," Microsystem Technologies, vol. 23, no. 8, pp. 3775-3789, 2017.

[28] H. Shi, Y. Kim, and Y. She, "Design of a parallel kinematic MEMS XY nanopositioner," in Proceedings of 2015 IEEE Conference on Robotics and Biomimetics, pp. 1973-1978, Zhuhai, China, 2015.

[29] X. Xi, T. Clancy, X. Wu, Y. Sun, and X. Liu, "A MEMS XYstage integrating compliant mechanism for nanopositioning at sub-nanometer resolution," Journal of Micromechanics and Microengineering, vol. 26, no. 2, article 025014, 2016.

[30] Z. Zhang, W. Zhang, Q. Wu et al., "Closed-form modelling and design analysis of $\mathrm{V}$ - and Z-shaped electrothermal microactuators," Journal of Micromechanics and Microengineering, vol. 27, no. 1, article 015023, 2017.

[31] Z. Zhang, Y. Yu, X. Liu, and X. Zhang, "A Comparison model of V- and Z-shaped electrothermal microactuators," in Proceedings of 2015 IEEE International Conference on Mechatronics and Automation, pp. 1025-1030, Beijing, China, August 2015.

[32] A. G. Fowler, M. Rakotondrabe, and S. O. R. Moheimani, "Closed-loop control of a novel 2-DOF MEMS nanopositioner with electrothermal actuation," in Proceedings of 6th IFAC Symposium on Mechatronic Systems, pp. 391-398, Hangzhou, China, 2013.

[33] Y. Zhu, S. O. R. Moheimani, and M. R. Yuce, "Bidirectional electrothermal actuator with Z-shaped beams," IEEE Sensors Journal, vol. 12, no. 7, pp. 2508-2509, 2012.

[34] E. T. Enikov, S. S. Kedar, and K. V. Lazarov, "Analytical model for analysis and design of V-shaped thermal microactuators," Journal of Microelectromechanical Systems, vol. 14, no. 4, pp. 788-798, 2005.

[35] A. M. H. Kwan, S. Song, X. Lu et al., "Improved designs for an electrothermal in-plane microactuator," Journal of Microelectromechanical Systems, vol. 21, no. 3, pp. 586-595, 2012.

[36] M. S. Suen, J. C. Hsieh, K. C. Liu, and D. T. W. Lin, “Optimal design of the electrothermal V-beam microactuator based on GA for stress concentration analysis," in Proceedings of the International Multiconference of Engineers and Computer Scientists, Hong Kong, March 2011.

[37] P. H. Godoy and E. C. N. Silva, "Aspects of fabrication and characterization of electro-thermal micro-actuators," Journal 
of the Brazilian Society of Mechanical Science and Engineering, vol. 29, no. 1, pp. 26-33, 2007.

[38] Z. Zhang, Y. Yu, X. Liu, and X. Zhang, "Dynamic electrothermal modeling of $\mathrm{V}$ - and Z-shaped electrothermal microactuator," in Proceedings of 2016 IEEE International Conference on Mechatronics and Automation, pp. 890-895, Harbin, China, August 2016.

[39] M. Burnie, "Modal analysis of MEMS gyroscopic sensors," Thesis, Department of Mechanical and Materials Engineering, Queen's University, Kingston, ON, Canada, 2010.

[40] W. F. Stokey, "Vibration of systems having distributed mass and elasticity," in Harris' Shock and Vibration Handbook, McGraw Hill Professional, New York, NY, USA, 2002.

[41] Z. Zhang, Y. Yu, and X. Zhang, "Vibration analysis of V-shaped beam electrothermal microactuators," in Proceedings of ASME 2017 International Design Engineering Technical Conferences and Computers and Information in Engineering Conference, IDETC/CIE 2017, Cleveland, Ohio, USA, August 2017.

[42] X. Shen and X. Chen, "Mechanical performance of a cascaded V-shaped electrothermal actuator," International Journal of Advanced Robotic Systems, vol. 10, no. 11, p. 379, 2013.

[43] A. Ali, R. A. Azim, U. S. Khan, A. A. Syed, and U. lzhar, "Design, simulation and optimization of electrothermal micro actuator," Applied Mechanics and Materials, vol. 229-231, pp. 1939-1943, 2012.

[44] J. Ouyang, M. McDonald, and Y. Zhu, "Temperaturedependent material properties of Z-shaped MEMS thermal actuators made of single crystalline silicon," Journal of Micromechanics and Microengineering, vol. 23, no. 12, article 125036, 2013.

[45] J. Chu, R. Zhang, and Z. Chen, “A novel SU-8 electrothermal microgripper based on the type synthesis of the kinematic chain method and the stiffness matrix method," Journal of Micromechanics and Microengineering, vol. 21, no. 5, article 054030, 2011. 


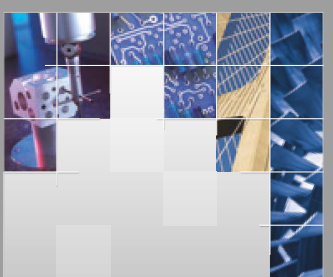

\section{Enfincering}
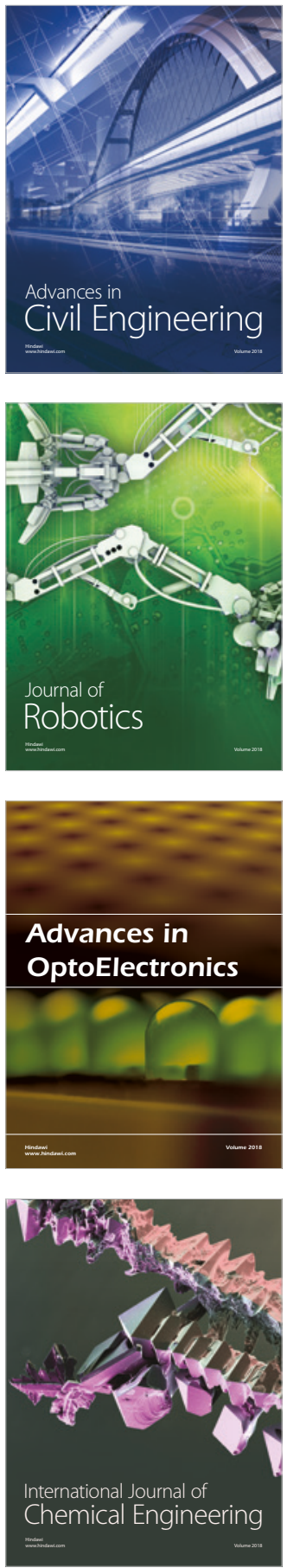

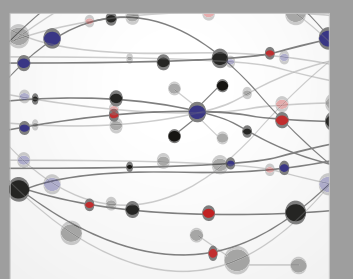

\section{Rotating \\ Machinery}

The Scientific World Journal

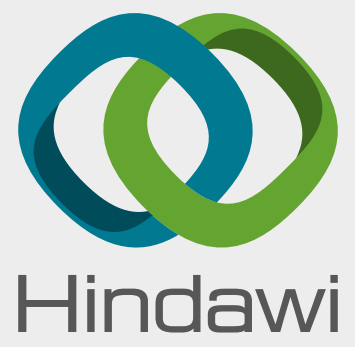

Submit your manuscripts at

www.hindawi.com
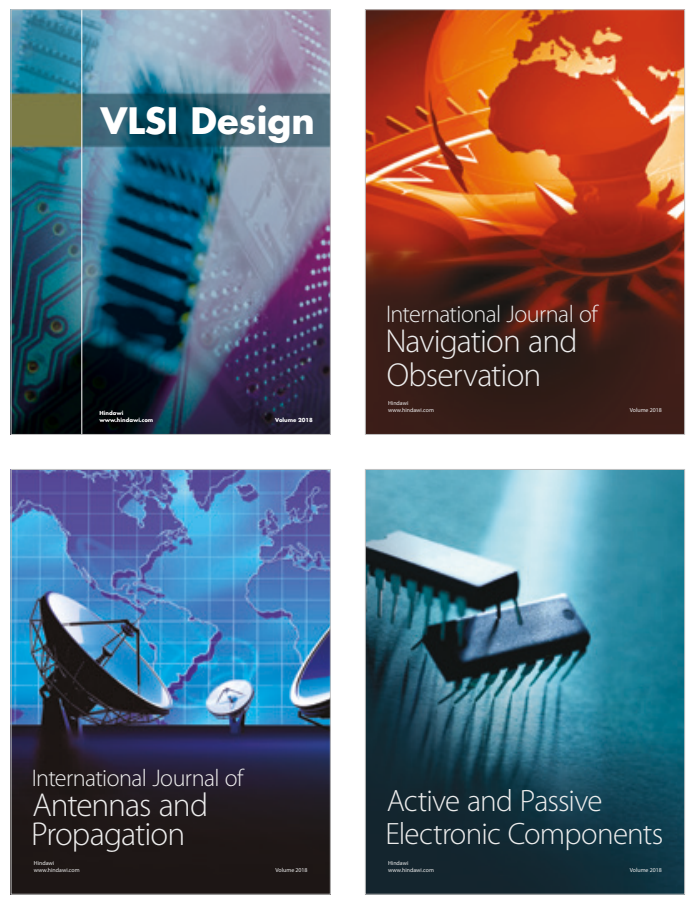
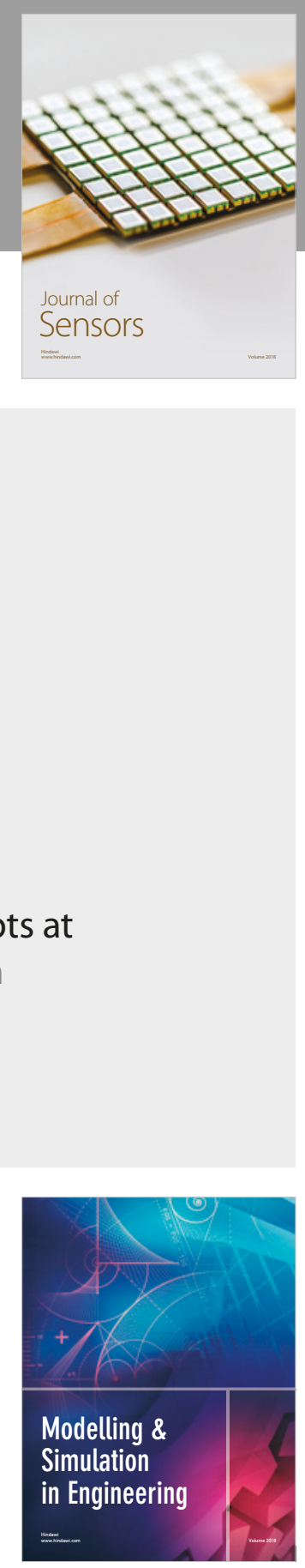

\section{Advances \\ Multimedia}
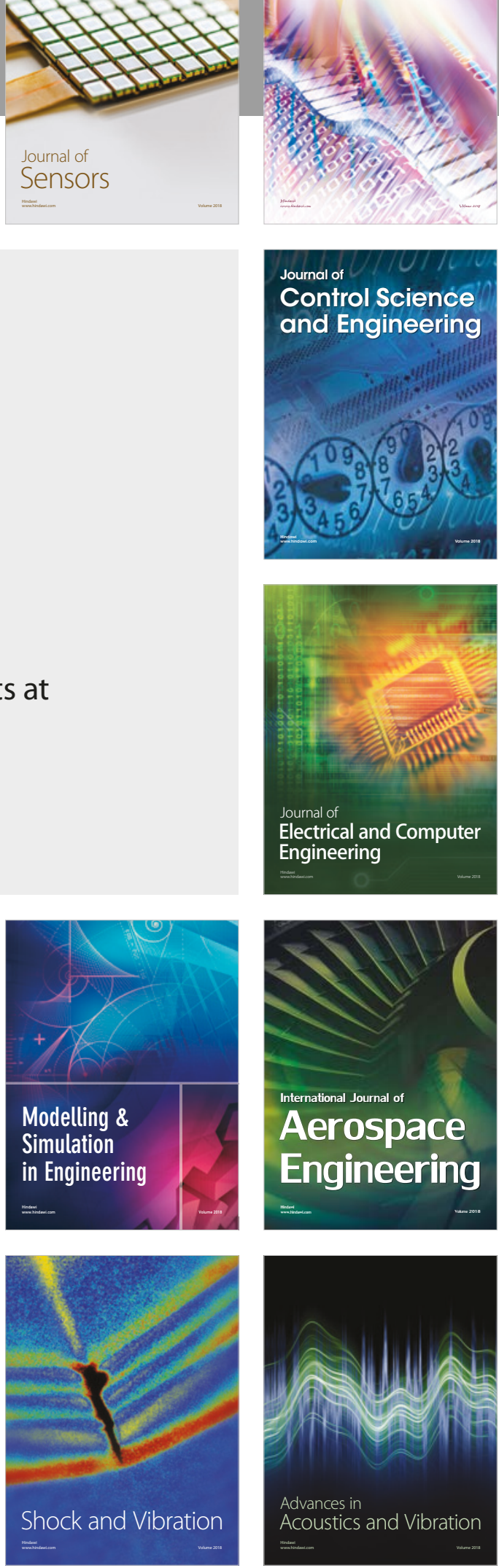